\title{
Utilizing in situ Electrochemical SHINERS for Oxygen Reduction Reaction Studies in Aprotic Electrolytes
}

Thomas A. Galloway, Laurence J. Hardwick*

*Stephenson Institute for Renewable Energy, Department of Chemistry, University of Liverpool, Liverpool, L69 7ZD

AUTHOR INFORMATION

Corresponding Author

* E-mail: hardwick@liverpool.ac.uk 


\section{Methods Section}

Chemicals. Tetrabutylammonium trifluoromethanesulfonate and tetrabutylammonium perchlorate (TBAOTF and $\mathrm{TBAClO}_{4}$ ), dimethyl sulfoxide (DMSO) were purchased from Sigma Aldrich. The salts were dried under vacuum overnight at $90^{\circ} \mathrm{C}$ and stored in an Argon filled glovebox before use. DMSO was distilled to remove impurities then stored over freshly activated molecular sieves for 1 week prior to use.

Synthesizing Au nanoparticles. $55 \mathrm{~nm} \mathrm{Au}$ nanoparticles were synthesized using the standard sodium citrate reduction method, ${ }^{1}$ trisodium citrate dihydrate (Aldrich) was added to a refluxing solution of chloroauric acid $\left(\mathrm{HAuCl}_{4}\right)($ Aldrich) while stirring and left to reflux for 40 minutes.

SHIN's (shell isolated nanoparticles) ${ }^{2}$. The gold nanoparticles were coated with a 2-3 nm silica shell. $0.4 \mathrm{ml}$ ((3-Aminopropyl)trimethoxysilane, 97\%) APTMS solution (1 mM) was added to $30 \mathrm{ml}$ Au nanoparticles while stirring. $3.2 \mathrm{ml}$ sodium silicate solution ( $\mathrm{pH}$ 10.2) was added. The solution was heated and stirred vigorously for 30 minutes. The solution was then washed multiple times and left in a water suspension. Before synthesis of SHIN's and Au nanoparticles all glassware was thoroughly cleaned with piranha solution to remove any impurities.

Electrochemical Measurements. Electrochemical experiments were conducted using a potentiostat (Biologic) in an argon glovebox using a specially designed, airtight 3 electrode glass cell, with a gas inlet and outlet for purging and bubbling of gases (Fig. S1.5). Specially dried oxygen and argon gas lines were plumbed directly into the glove box to allow for bubbling and purging of the electrolyte, leading to minimal $\mathrm{H}_{2} \mathrm{O}$ contamination $<20 \mathrm{ppm}$. Various $3 \mathrm{~mm}$ diameter working disc electrodes were used (GC, Au, Pt). A Pt coil was used as the counter electrode and an Ag wire was used as the reference electrode. The reference electrode was standardized using ferrocene after the experiments, and calibrated against either the $\mathrm{Ag} / \mathrm{Ag}^{+}$or the $\mathrm{Li} / \mathrm{Li}^{+}$couple. Between experiments all apparatus was cleaned and sonicated with Milli-Q water and acetone. The working electrodes were cleaned using 
acetone and Milli-Q water then polished using $1 \mu \mathrm{m}, 0.3 \mu \mathrm{m}$ and $0.05 \mu \mathrm{m}$ alumina suspensions to produce a smooth surface. Everything was dried under vacuum before use.

Characterization Measurements. Raman experiments were carried out on a Renishaw In-Via Raman spectrometer with an inverted microscope using a $785 \mathrm{~nm}$ wavelength laser. A specifically designed airtight, three electrode cell with a sapphire window at the bottom of the cell was used for the Raman experiments. The cell was assembled in an argon filled glovebox, the electrolyte was purged with Ar to remove any gases, and then bubbled with oxygen for 30 minutes to saturate the electrolyte, before being sealed and transferred to the Raman spectrometer.

SERS. An electrochemical roughened $\mathrm{Au}$ disc electrode $(0.16 \mathrm{~mm})$ was used as the working electrode. The working electrode was roughened, using oxidation and reduction cycles in $0.1 \mathrm{M} \mathrm{KCl}$ solution and dried under vacuum prior to use as described previously. ${ }^{3}$

SHINERS. $2 \mu 1$ of SHIN's were drop cast onto a smooth electrode surface. The electrode was left to dry in a stream of nitrogen prior to use. Before all experiments the apparatus was cleaned and sonicated with Milli-Q water and acetone and dried under vacuum. All glassware was acid washed before use.

TEM. A JEOL 3010 transmission electron microscope was used for the collection of TEM images. 


\section{Fig S1. Characterizing SHINERS ${ }^{4}$}

\section{$\underline{\text { Tests for pinholes }}$}

$\underline{\text { TEM }}$

TEM can be used as a visual aid for large pinholes (Figure S1.1). If pinholes are small they may not be visible in TEM. Cyclic voltammetry and Raman spectroscopy are both very good experimental techniques to determine if any pinholes are present.
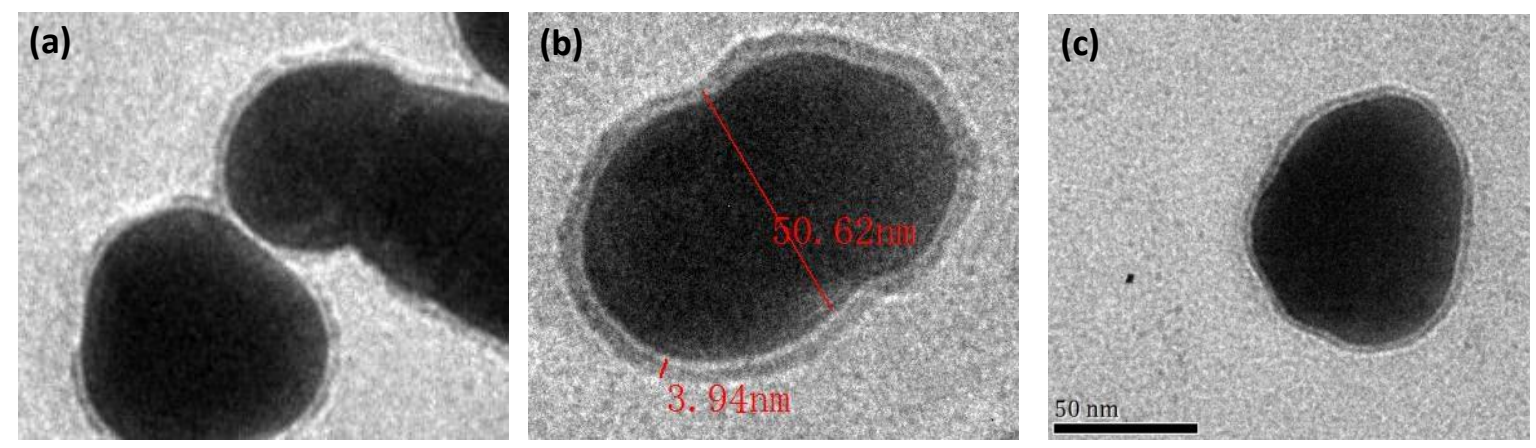

Figure S1.1 TEM images of a shell isolated nanoparticle, dark centre is the Au core surrounded by a light grey $\mathrm{SiO}_{2}$ shell (a) $\mathrm{SiO}_{2}$ shell $<2 \mathrm{~nm}$ with pinholes (b) $\mathrm{SiO}_{2}$ shell $4 \mathrm{~nm}\left(\right.$ c) $\mathrm{SiO}_{2}$ shell 2-3 nm. 


\section{Cyclic voltammetry}

$2 \mu \mathrm{l}$ of SHINERS are deposited on a glassy carbon electrode and left to dry in the atmosphere. A cyclic voltammogram was run in a $0.1 \mathrm{M} \mathrm{H}_{2} \mathrm{SO}_{4}$ electrolyte (Figure S1.2). If pinholes are present peaks at 0.9 $\mathrm{V}$ in the reduction scan and $1.2 \mathrm{~V}$ in the oxidation scan will be present. If no peaks are present there is no oxidation or reduction of Au indicating a completely inert shell.

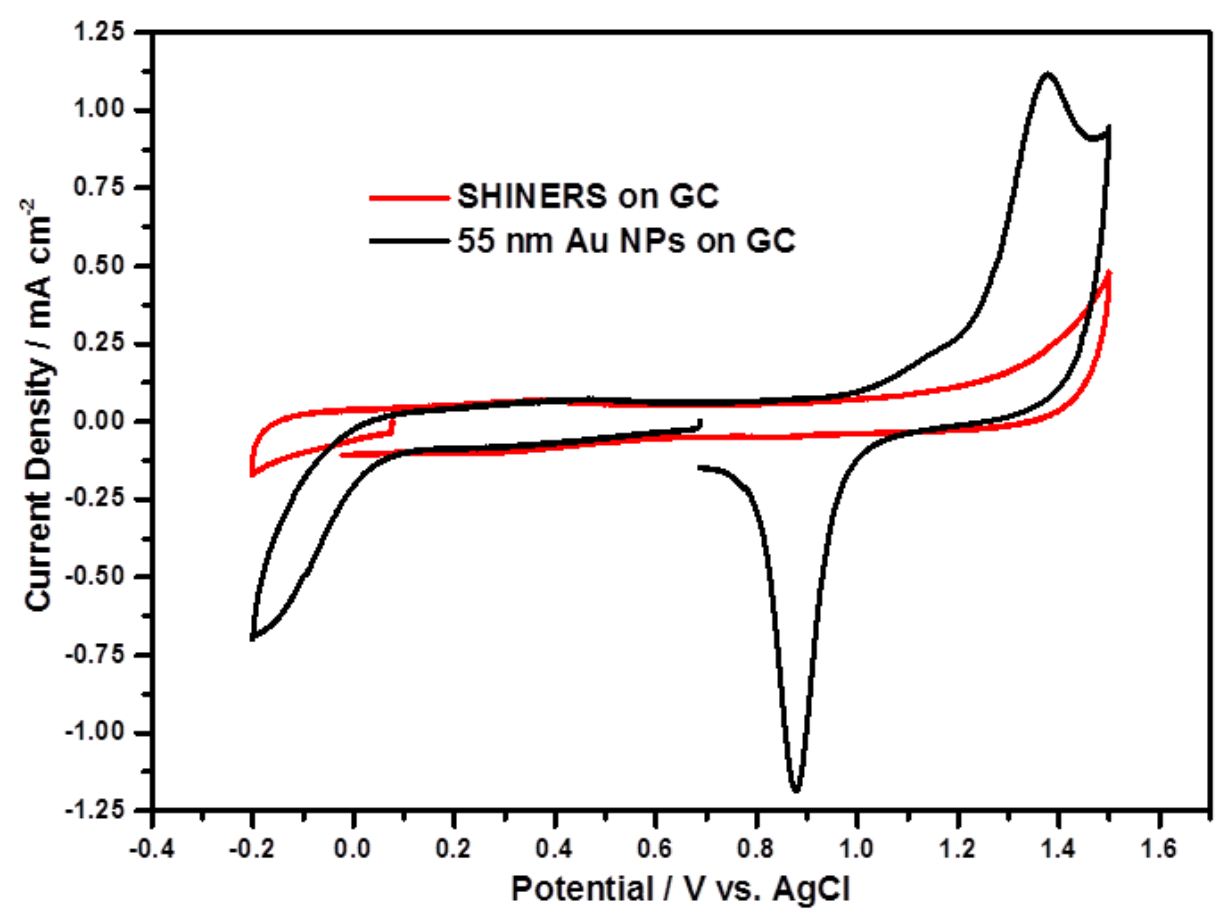

Figure S1.2 Cyclic voltammograms of SHINERS (red) and Au nanoparticles (black) on a glassy carbon electrode in $0.1 \mathrm{M} \mathrm{H}_{2} \mathrm{SO}_{4}$. Sweep rate $10 \mathrm{mv} \mathrm{s}^{-1}$. 


\section{Raman spectroscopy}

Raman spectroscopy is used to check the silica shell for pinholes. SHINERS are absorbed onto the GC surface; pyridine is then dropped onto to the surface and left to adsorb. If pinholes are present 2 peaks will be visible in the Raman spectrum at 1010 and $1030 \mathrm{~cm}^{-1}$ (Figure S1.3a.). If no peaks are present pyridine has not adsorbed onto the Au surface, therefore there are no pinholes (Figure S1.3b.).

The enhancement factor of SHINERS is dependent on the shell thickness; Raman spectroscopy can be used to calculate this. SHINERS are deposited on an Au surface and pyridine is dropped on and left to adsorb. The greatest enhancement is observed for SHINERS with a shell thickness of 2-3 nm (Figure S1.3c). If the shell is too thick the enhancement will be drastically reduced (Figure S1.3d.)

(c)

Au NP

(a)

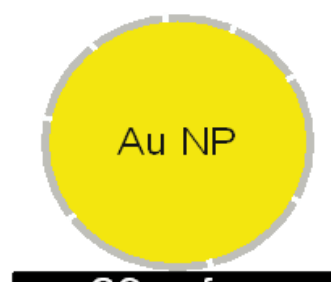

GC surface

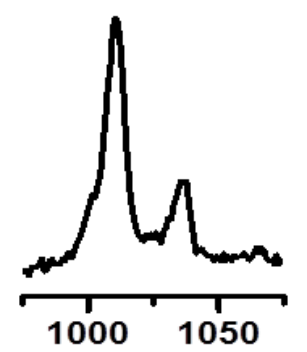

(b)

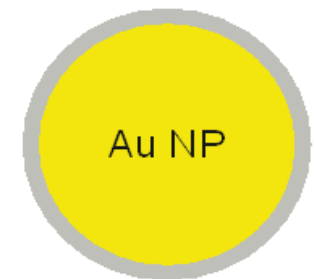

GC surface

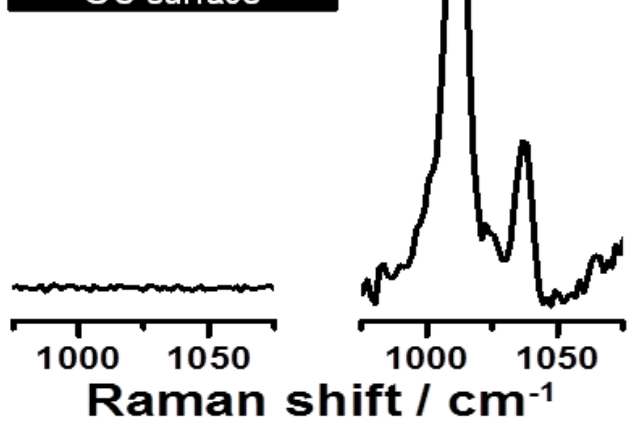

(d)
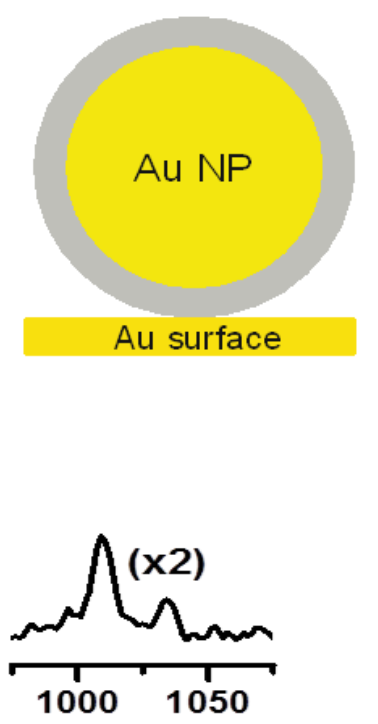

Figure S1.3 Raman spectrums of pyridine on (a) GC/ SHINERS with pinholes (b) GC/ SHINERS without pinholes (c) Au/ SHINERS (2 nm shell) (d) Au/SHINERS (4 nm shell). 
Refering to the Raman spectrum (3-aminopropyl)trimethoxysilane (APTMS) (Figure S1.4 and the tabulation of main bands (Table S1) no bands APTMS were detected on SHINERS particles or during SHINERS studies as discussed by Smith et $a l^{5}$

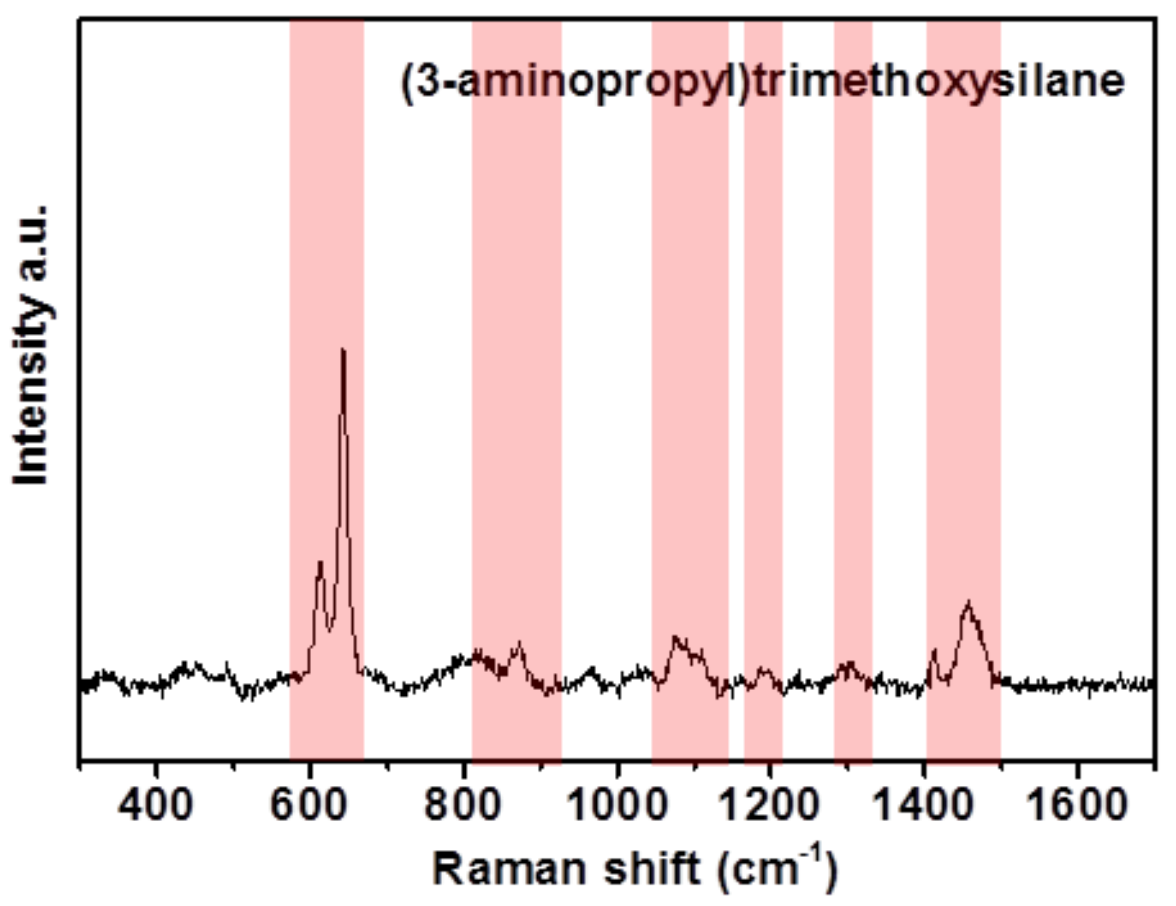

Figure S1.4 Raman spectrum of (3-aminopropyl)trimethoxysilane (APTMS).

Table S1 Peak positions and assignments of APTMS Raman bands.

\begin{tabular}{|c|c|}
\hline Peak Position $\left(\mathbf{c m}^{-\mathbf{1}}\right)$ & Assignment \\
\hline 440 & $\mathrm{Si}\left(\mathrm{OCH}_{3}\right)_{3}$ \\
\hline 612 & $\mathrm{Si}-\mathrm{CH}_{2} \mathrm{R}$ \\
\hline 643 & $\mathrm{SiO}-\mathrm{CH}_{3}$ \\
\hline 800 & $\mathrm{OSi}^{-} \mathrm{CH}_{2} \mathrm{R}$ \\
\hline 873 & $\mathrm{Si}-\mathrm{OC}$ \\
\hline 1076 & $\mathrm{H}_{2} \mathrm{C}-\mathrm{NH}_{2}$ \\
\hline 1104 & $\mathrm{SiO}-\mathrm{C}$ \\
\hline 1187 & $\mathrm{SiO}-\mathrm{CH}_{3}$ \\
\hline 1305 & $\mathrm{Si}-\mathrm{OCH}_{3}$ \\
\hline 1413 & $\mathrm{O}_{-} \mathrm{CH}_{3}$ \\
\hline 1457 & $\mathrm{CNH}_{2}$ \\
\hline
\end{tabular}



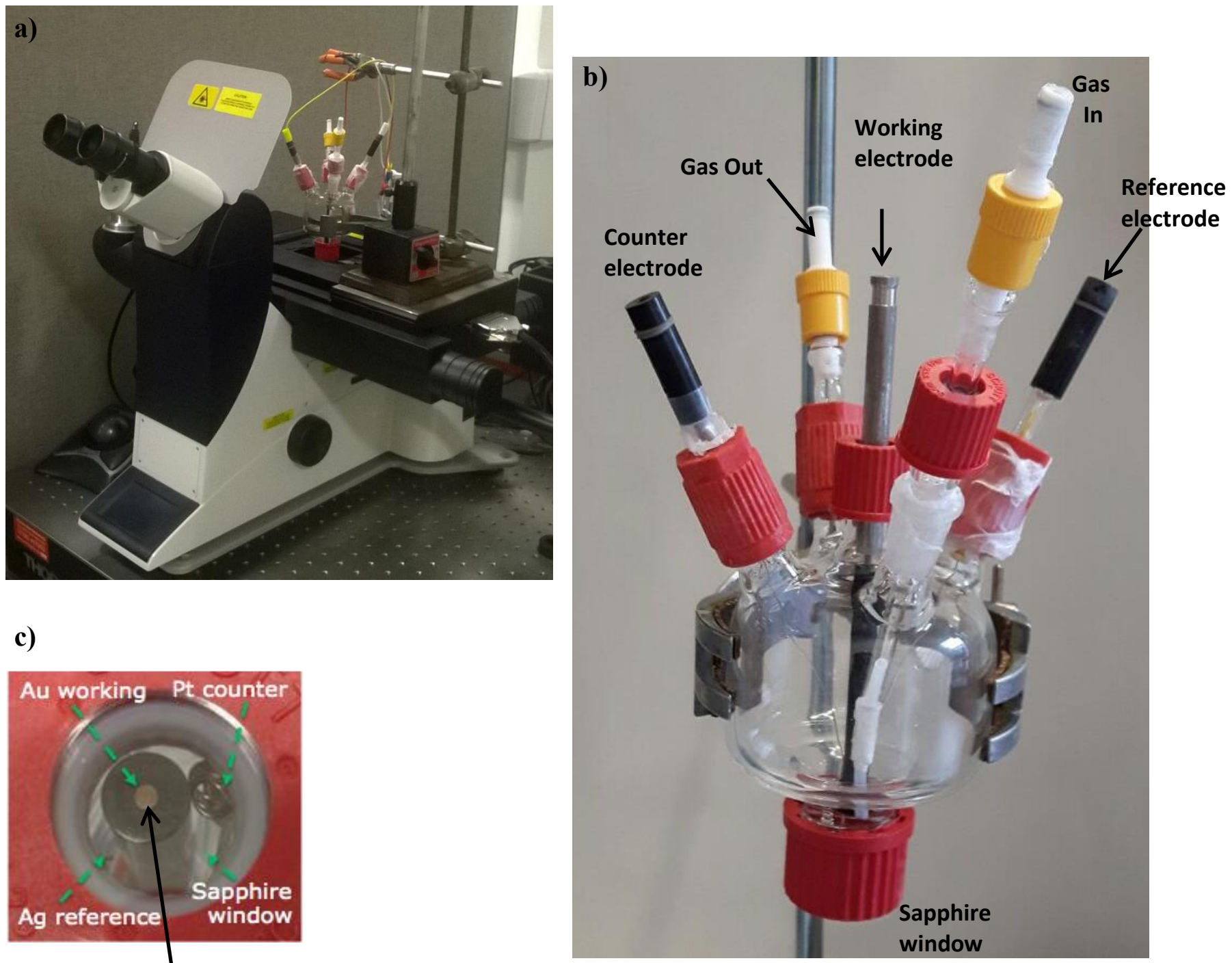

c)
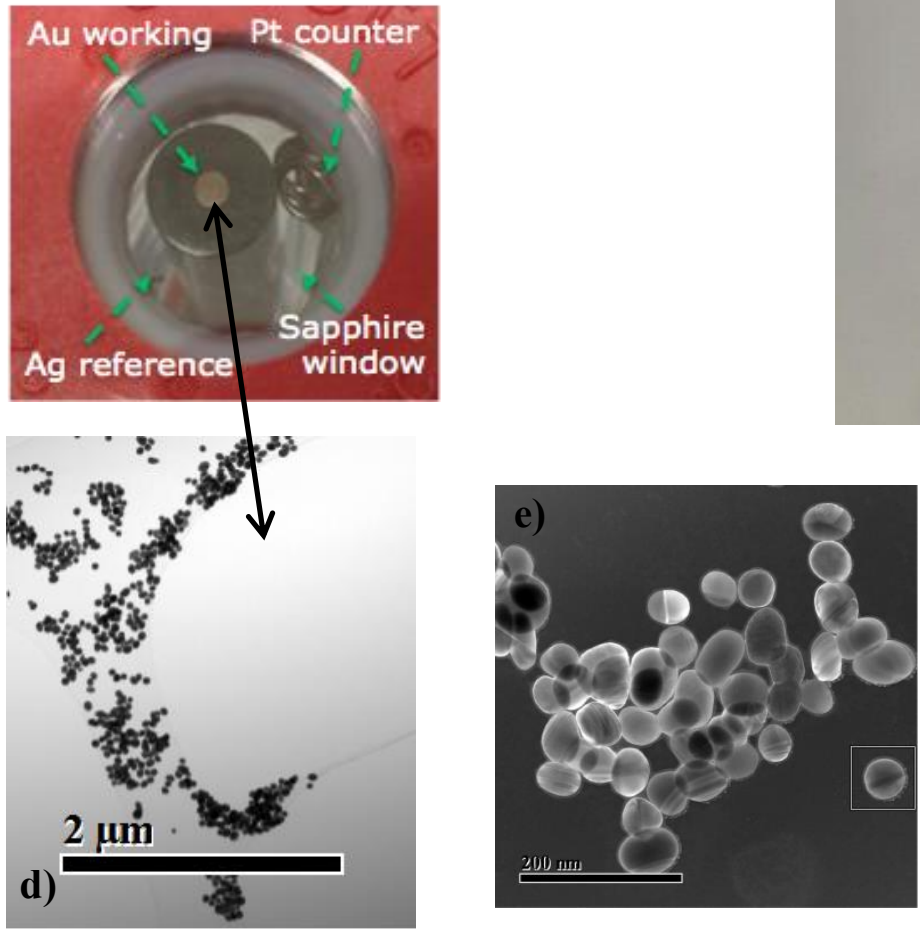

Figure S1.5 a) Electrochemical cell setup on inverted microscope b) Electrode setup in specially designed glass cell c) View through sapphire window on the bottom of the cell d) TEM showing SHINERS distribution e) Higher magnification dark field TEM image of SHINERS. 


\section{Calculating the Enhancement factor $(G)^{6}$}

The enhancement factor can vary slightly from surface to surface due to the roughness factor which has to be accounted for, but there is no plasmon coupling between the SHINS and the electrode surface

$$
\text { Eq 1. } \quad G=\frac{\left(I_{\text {surface }} / N_{\text {surface }}\right)}{\left(I_{\text {solution }} / N_{\text {solution }}\right)}
$$

$\mathbf{I}_{\text {surface }}$ - Integrated intensity of the pyridine $\left(v_{1 \mathrm{a}}\right)$ ring breathing mode for surface species

$\mathbf{I}_{\text {bulk }}$ - Integrated intensity of the pyridine $\left(v_{1 \mathrm{a}}\right)$ ring breathing mode for solution species

$\mathbf{N}_{\text {solution }}$ - The number of solution molecules present in the laser beam

$\mathbf{N}_{\text {surface }}$ - The number of surface molecules present in the laser beam

$$
\text { Eq 2. } \quad \mathbf{N}_{\text {surface }}=\frac{\mathbf{R A}}{\boldsymbol{\sigma}} \quad \text { (assuming monolayer adsorption) }
$$

R - Roughness factor

A - Area of focal spot of the Raman laser

$\boldsymbol{\sigma}-$ Area of 1 absorbed pyridine molecule

$$
\text { Eq 3. } \quad \mathbf{N}_{\text {bulk }}=A \text { h c } \mathbf{N}_{\mathbf{a}}
$$

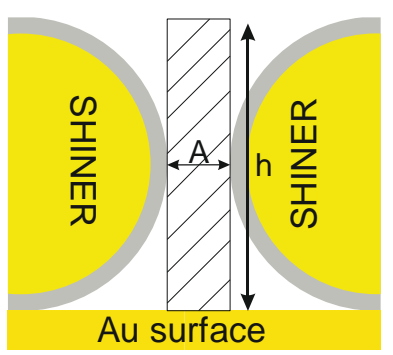

$\mathrm{h}-$ Focal depth of the laser

$\mathrm{c}$ - Concentration of pyridine

The enhancement factor can then be calculated from combining equations 1,2 and 3

$$
\text { Eq 4. } \quad G=\frac{I_{\text {surface }} c N_{\mathrm{a}} \sigma h}{I_{\text {sol }} R}
$$

Typical enhancement factors of up to $1.5 \times 10^{8}$ can be achieved for SHINERS on Au(111) surfaces ${ }^{7}$

We achieved an enhancement factor of $2.9 \times 10^{5}$ on polycrystalline gold electrode. This was calculated with the above equation using the pyridine adsorption on gold with SHINERS from Figure S1.3. The enhancement was not as strong as SERS, but still produced a suitable enhancement in order to detect products on the electrode surface. 
a)

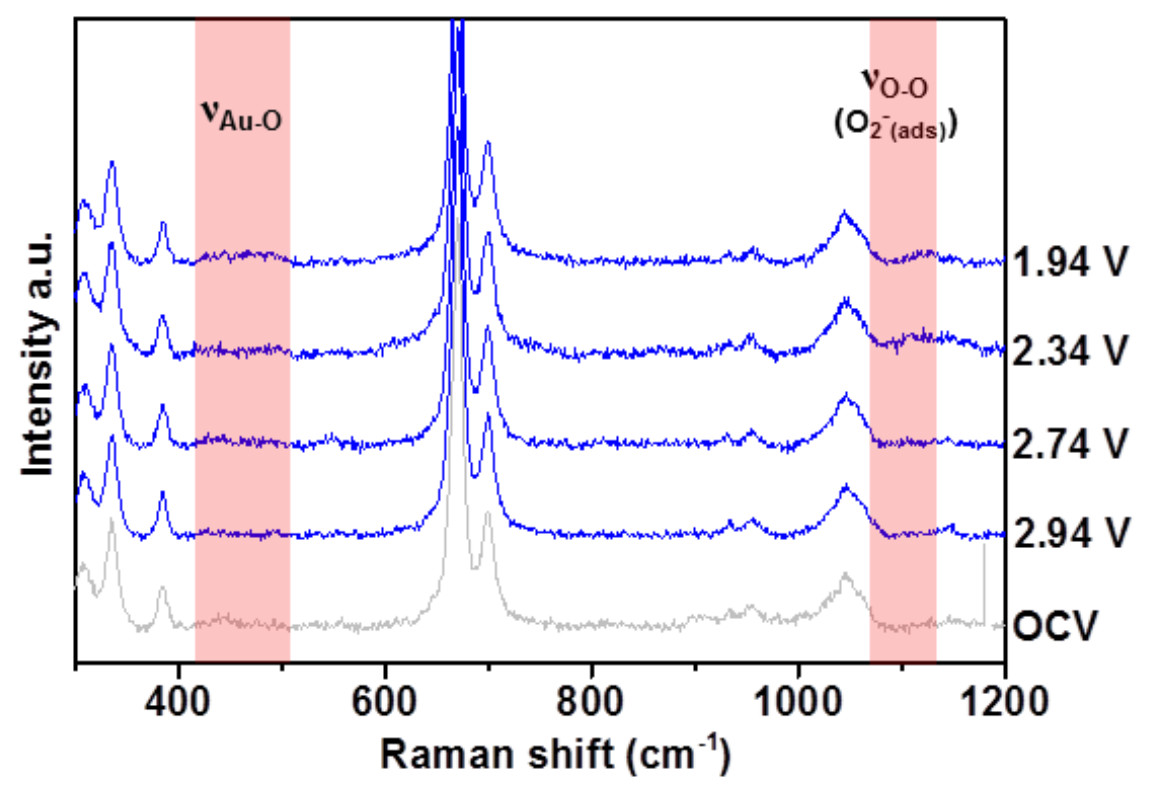

b)

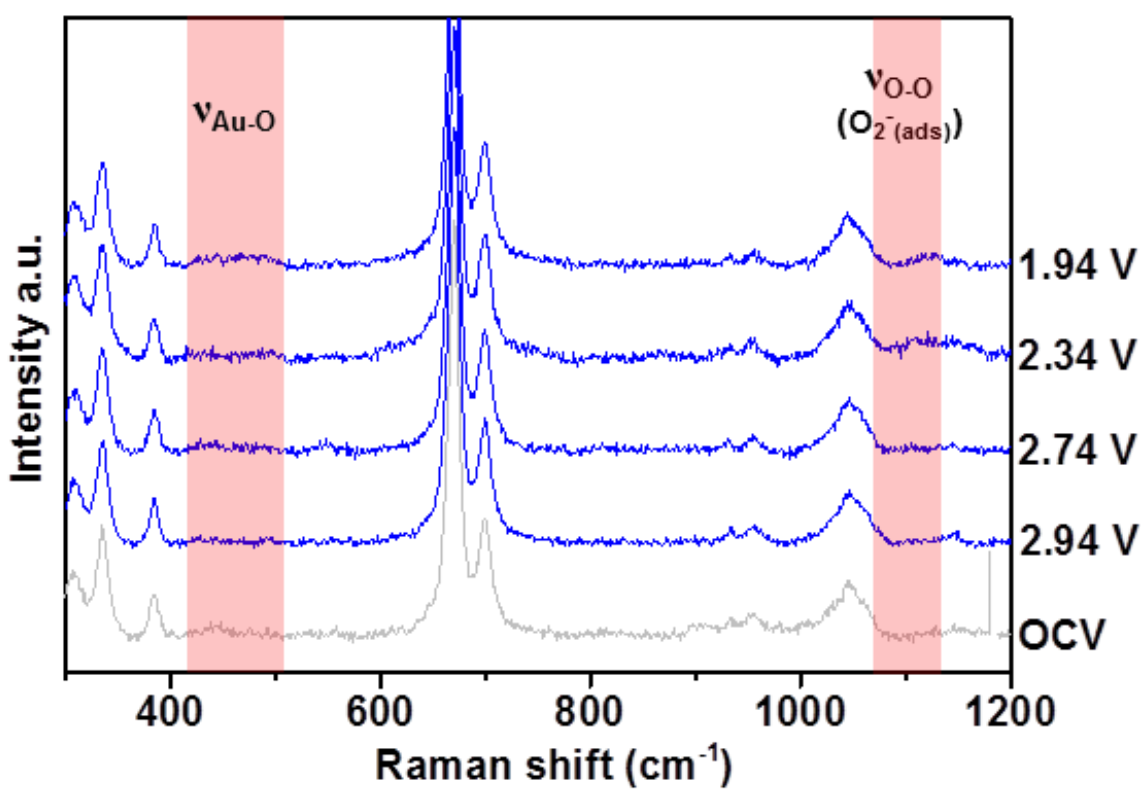

Figure S2 a) SERS b) SHINERS of 0.1M TBAClO 4 in DMSO (in argon, deoxygenated electrolyte).

The electrolyte was purged with argon for 30 minutes in order to remove any trace gases (including oxygen) from the electrolyte. In situ SERS was then carried out to track the ORR without the presence of oxygen. No peaks around $400-500 \mathrm{~cm}^{-1}$ or $1100 \mathrm{~cm}^{-1}$ were observed. This implied that these peaks only occur in the presence of oxygen. No potential dependent bands from the electrolyte were observed. 


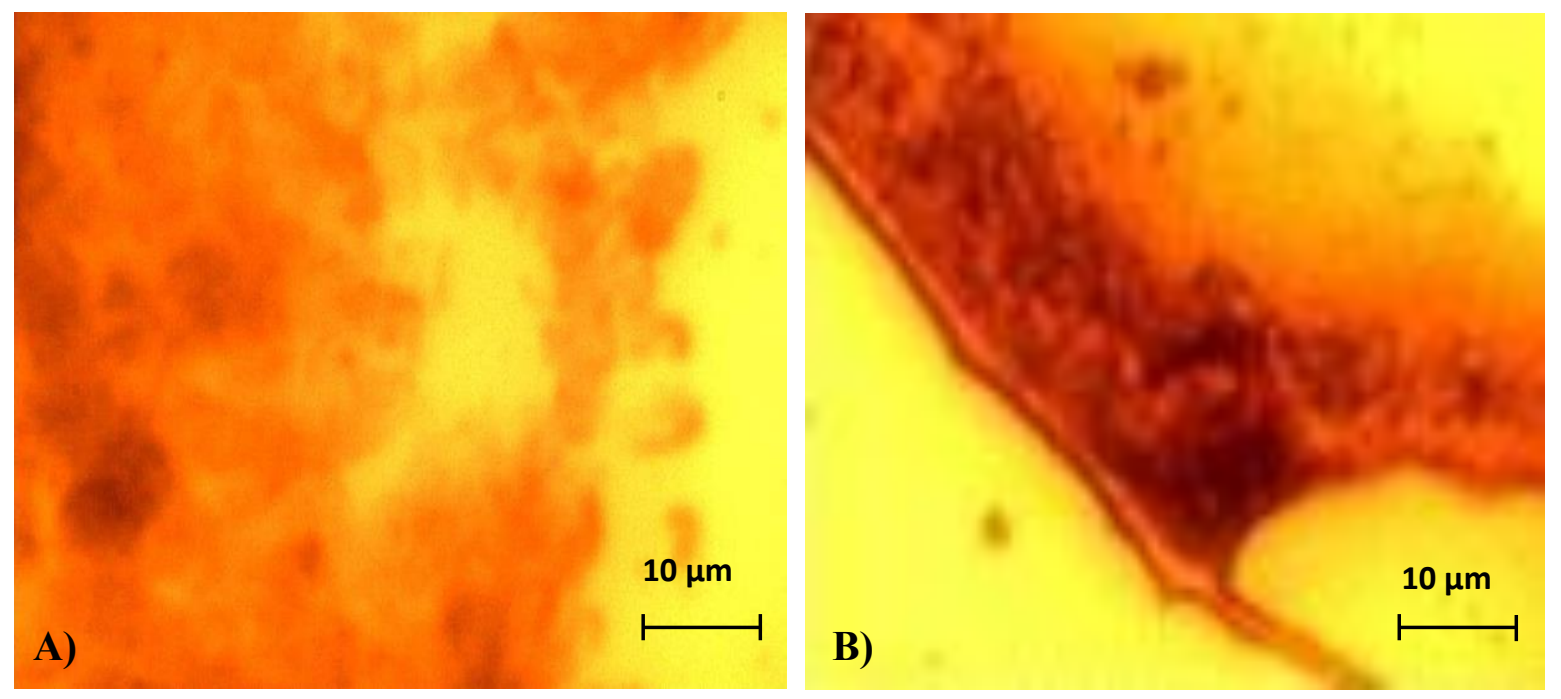

Figure S3 Microscope images taken with an in via optical microscope of SHINS dropcast onto a gold electrode surface. a) particles dried under vacuum b) dried in the atmosphere. Bright patches indicate gold electrode with no SHINERS particles, dark patches indicates presence of SHINERS particles upon the surface.

Depending on the method of drying the electrode a different degree of dispersion of the SHIN particles is obtained upon the electrode surface. The particles dried under vacuum have a much better dispersion than particles left to dry in atmosphere. Due to the cohesive nature of water the particles form a thick ring (Figure S2.b), whereas particles dried under vacuum have a much more even dispersion. A monolayer coverage is required to gain the greatest enhancement from the SHINERS technique. 


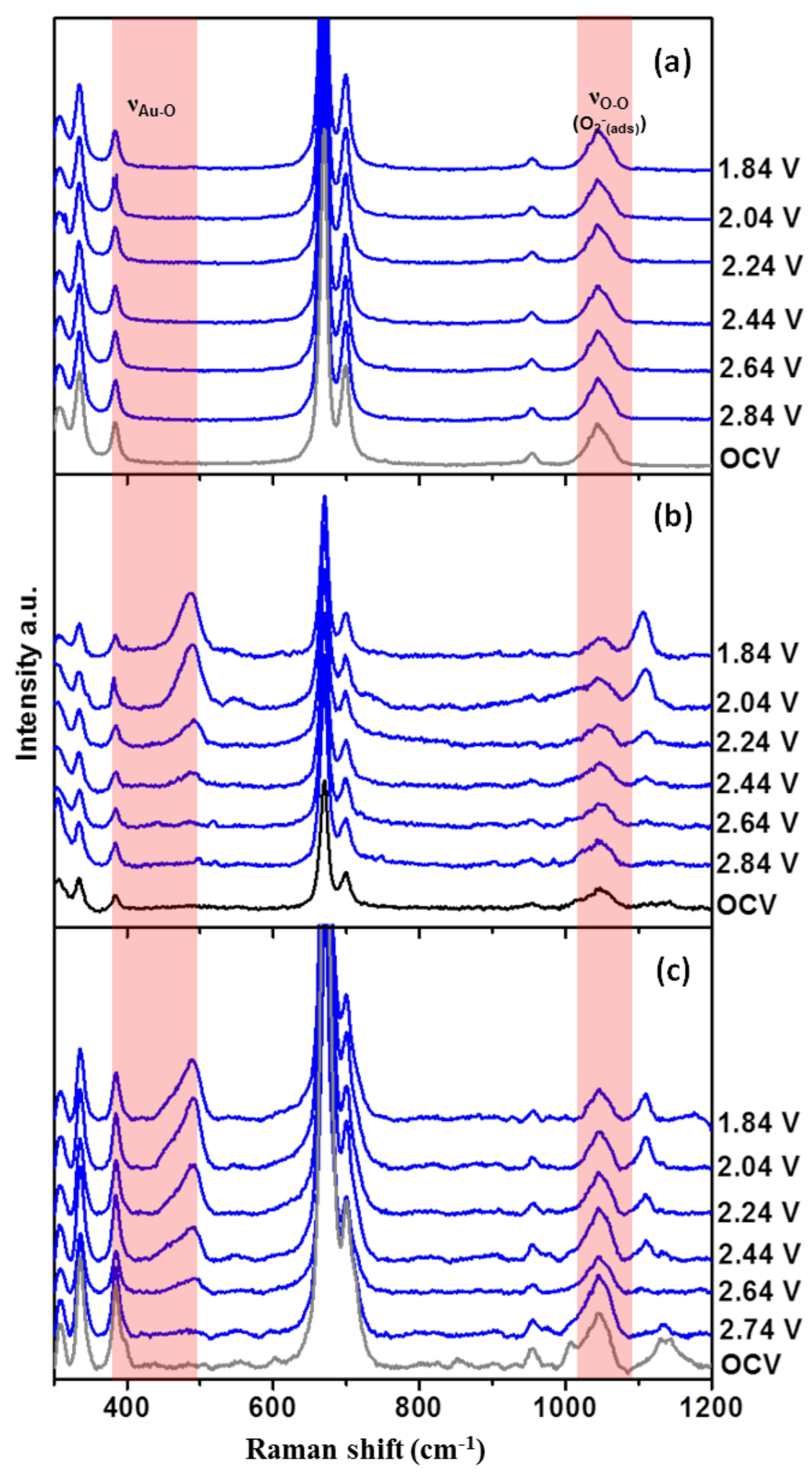

Figure S4 In situ Raman spectra of the ORR in 0.1 M TBAOTf/ DMSO electrolyte on (a) smooth (b) roughened (c) SHIN's drop cast on the surface) polycrystalline gold electrode. All potentials vs $\mathrm{Li} / \mathrm{Li}^{+}$. 


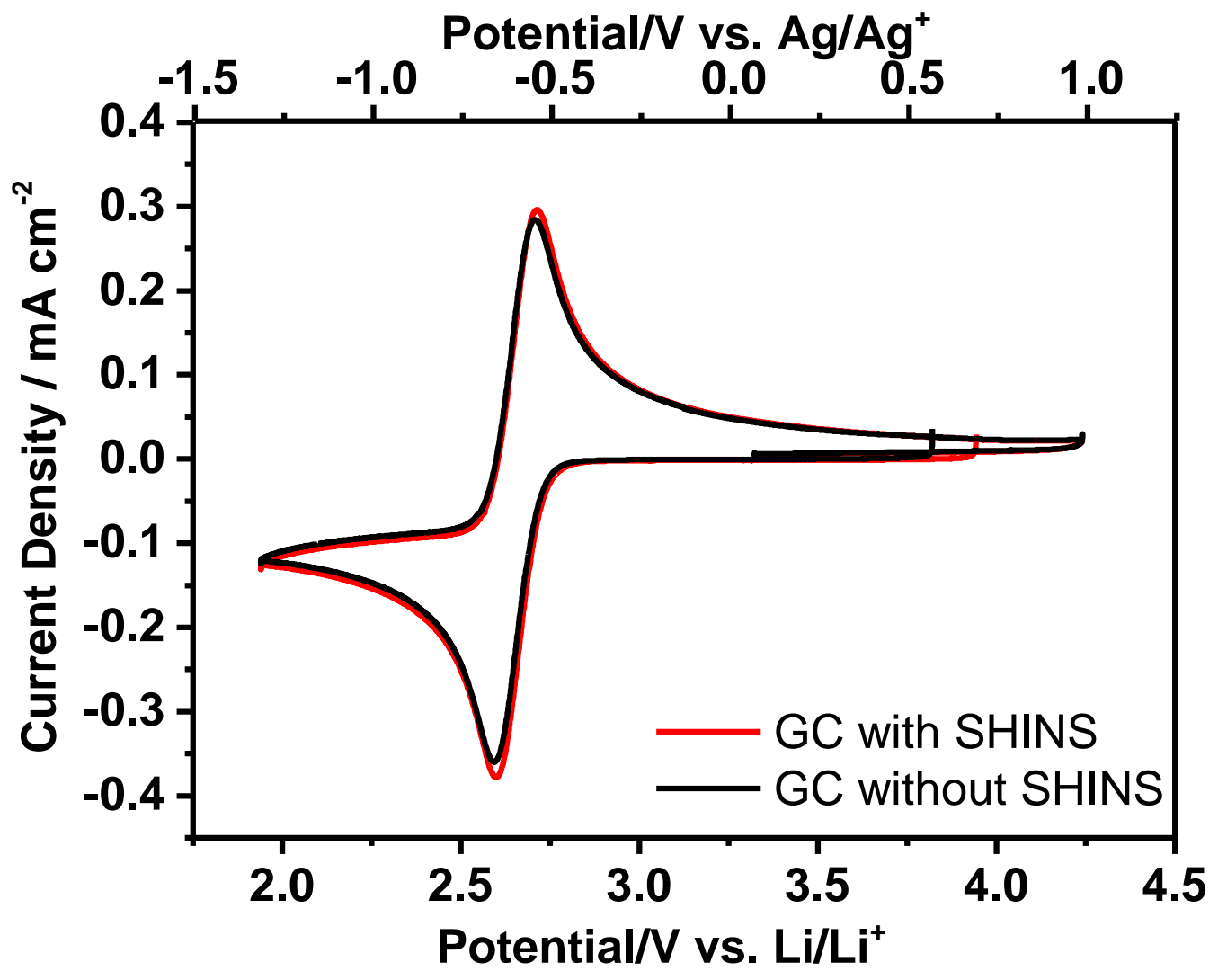

Figure S5 Cyclic voltamograms on GC electrode in $0.1 \mathrm{M} \mathrm{TBAClO}_{4} / \mathrm{DMSO}$ electrolyte with and without SHINERS particles deposited on the surface. 


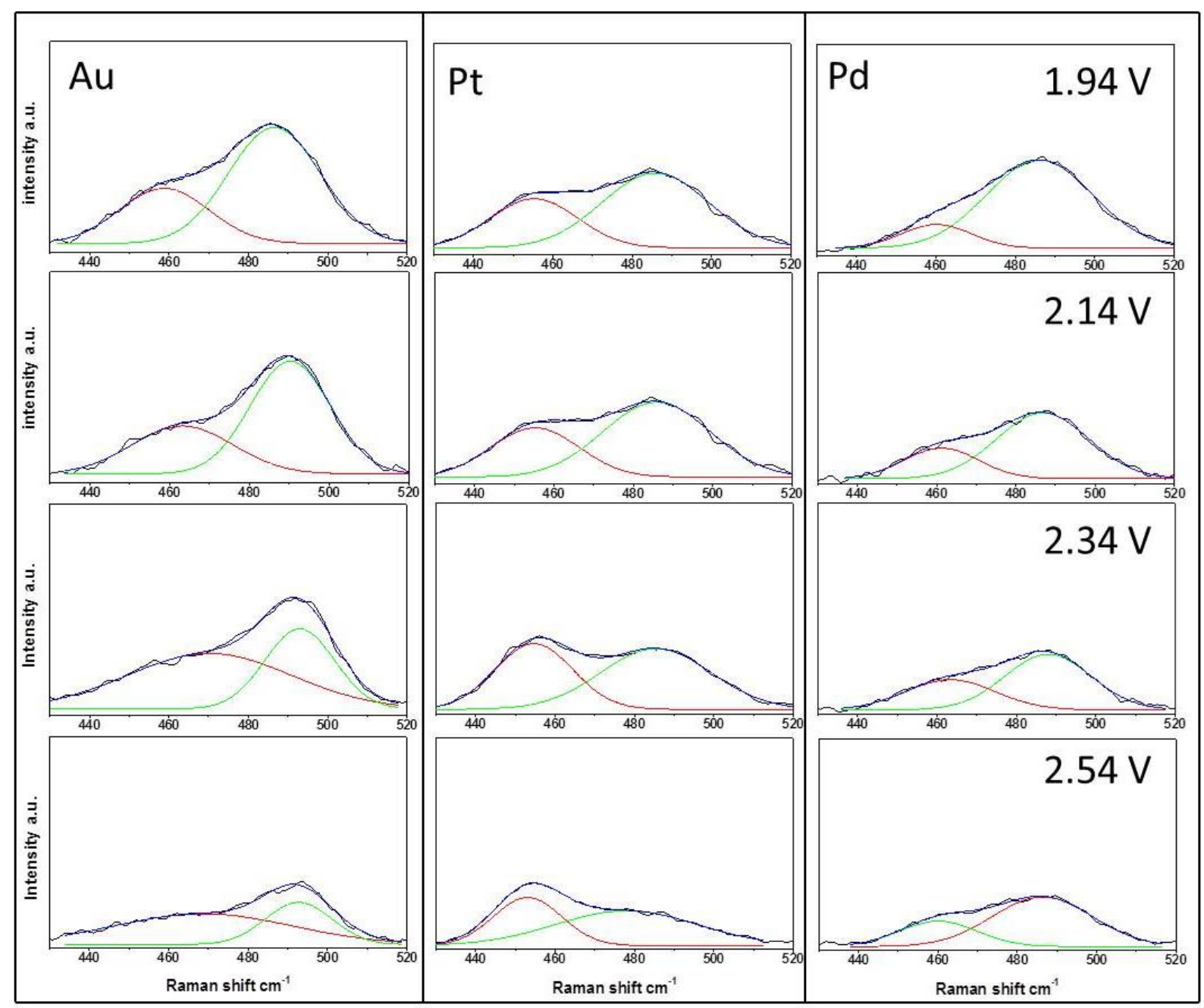

Figure S6 Raman spectrums with peak fitting to demonstrate the shift in peak position of the metaloxygen bond vibration with changing electrode surface.

Table S2 Corresponding peak positions and FWHM of peak fitted bands of the shift in peak position of the metal-oxygen bond vibration with alternative electrode surfaces.

\begin{tabular}{|c|c|c|c|c|c|c|}
\hline & \multicolumn{2}{|c|}{ Au } & \multicolumn{2}{|c|}{$\mathbf{P t}$} & \multicolumn{2}{|c|}{ Pd } \\
\hline Potential & $\begin{array}{c}\text { Peak Position } \\
\left(\mathrm{cm}^{-1}\right)\end{array}$ & $\begin{array}{c}\text { FWHM } \\
\left(\mathrm{cm}^{-1}\right)\end{array}$ & $\begin{array}{c}\text { Peak Position } \\
\left(\mathrm{cm}^{-1}\right)\end{array}$ & $\begin{array}{c}\text { FWHM } \\
\left(\mathbf{c m}^{-1}\right)\end{array}$ & $\begin{array}{c}\text { Peak Position } \\
\left(\mathrm{cm}^{-1}\right)\end{array}$ & $\begin{array}{c}\text { FWHM } \\
\left(\mathbf{c m}^{-1}\right)\end{array}$ \\
\hline \multirow{2}{*}{$1.94 \mathrm{~V}$} & 458 & 27 & 455 & 26 & 460 & 21 \\
\hline & 486 & 27 & 485 & 32 & 486 & 32 \\
\hline \multirow{2}{*}{$2.14 \mathrm{~V}$} & 461 & 30 & 455 & 26 & 461 & 22 \\
\hline & 490 & 24 & 485 & 32 & 486 & 27 \\
\hline \multirow{2}{*}{$2.34 \mathrm{~V}$} & 470 & 48 & 454 & 23 & 463 & 26 \\
\hline & 492 & 20 & 485 & 33 & 488 & 26 \\
\hline \multirow{2}{*}{$2.54 \mathrm{~V}$} & 468 & 53 & 453 & 20 & 460 & 33 \\
\hline & 493 & 20 & 477 & 42 & 486 & 30 \\
\hline
\end{tabular}




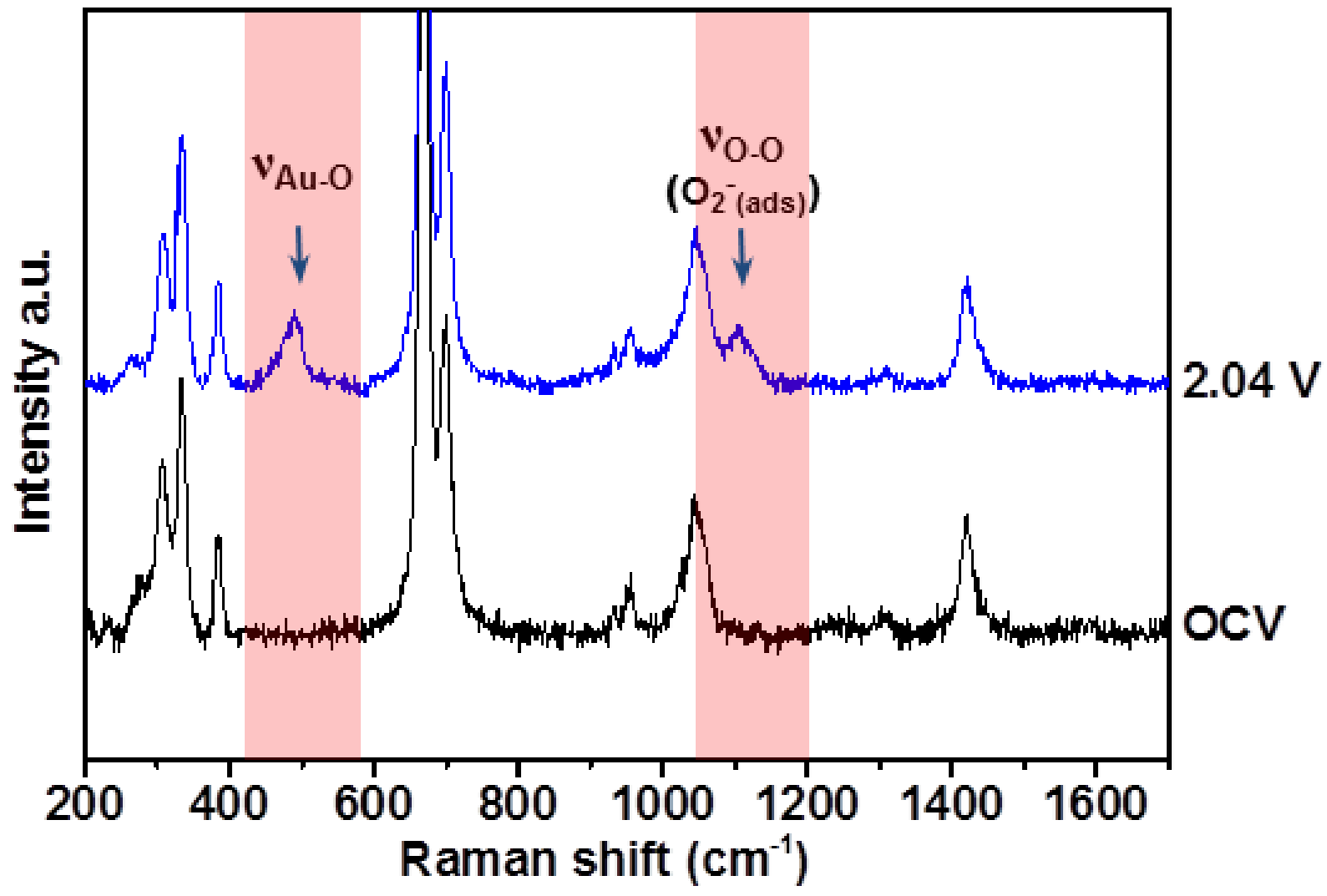

Figure S7 SHINERS spectra with wider spectral range for $0.1 \mathrm{M} \mathrm{TBAClO}{ }_{4} / \mathrm{DMSO}$ at OCV and $2.04 \mathrm{~V}$ on $\mathrm{Au}$. No new peaks were observed under potential control. 


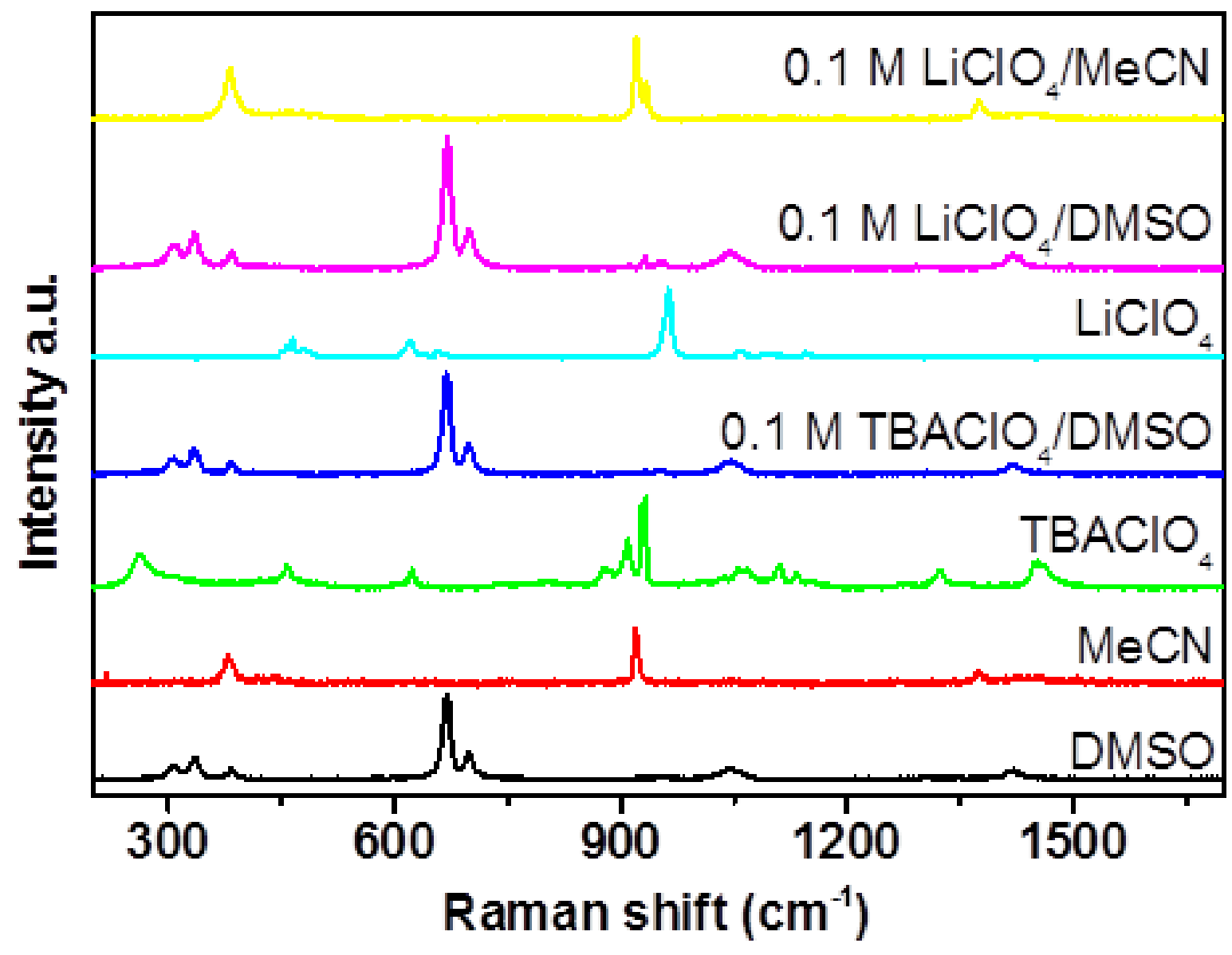

Figure S8 Standard Raman spectra for salts, solvents and electrolytes used in the experiments. 


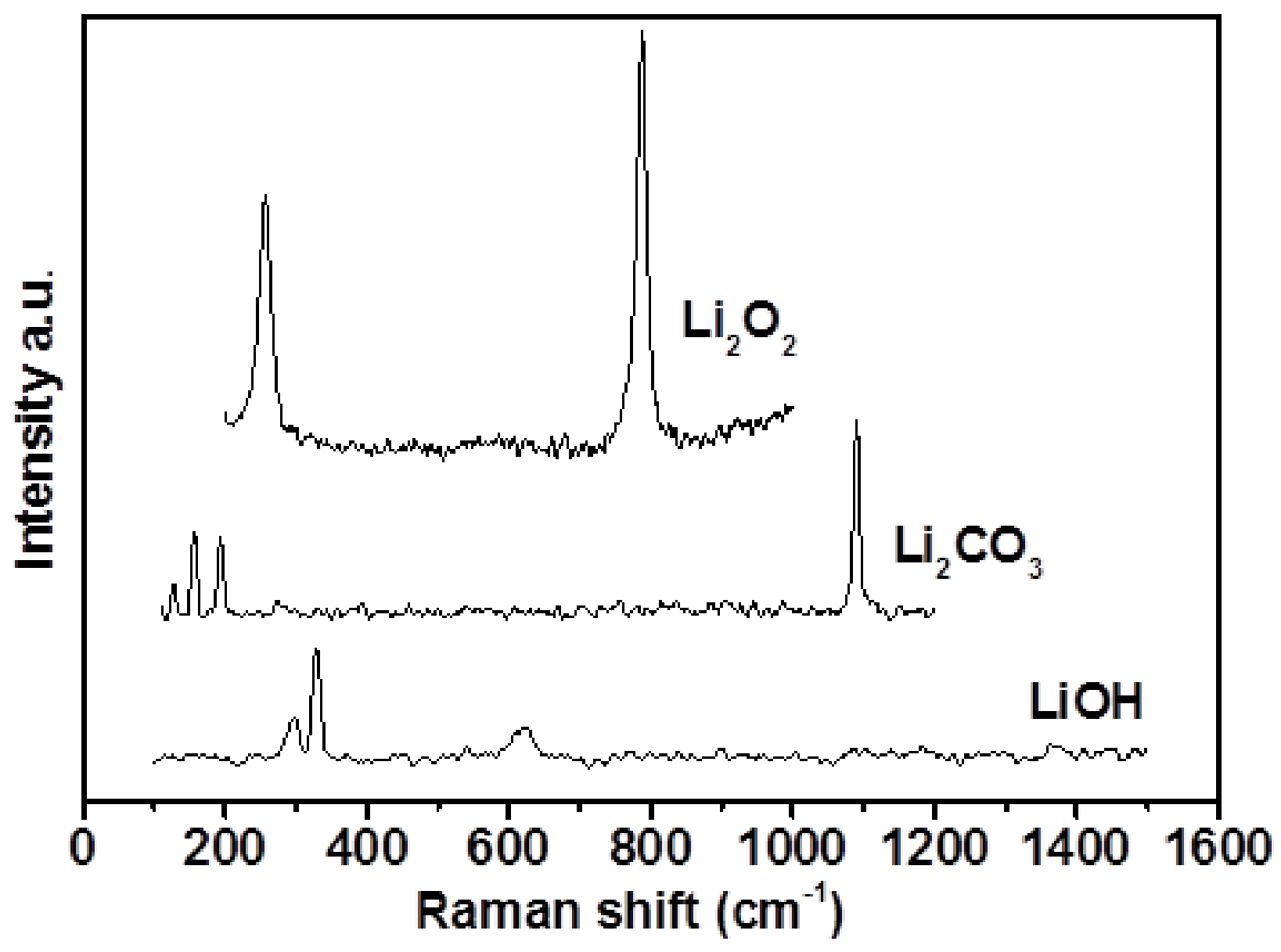

Figure S9 Standard Raman spectra for typical lithium salts. 


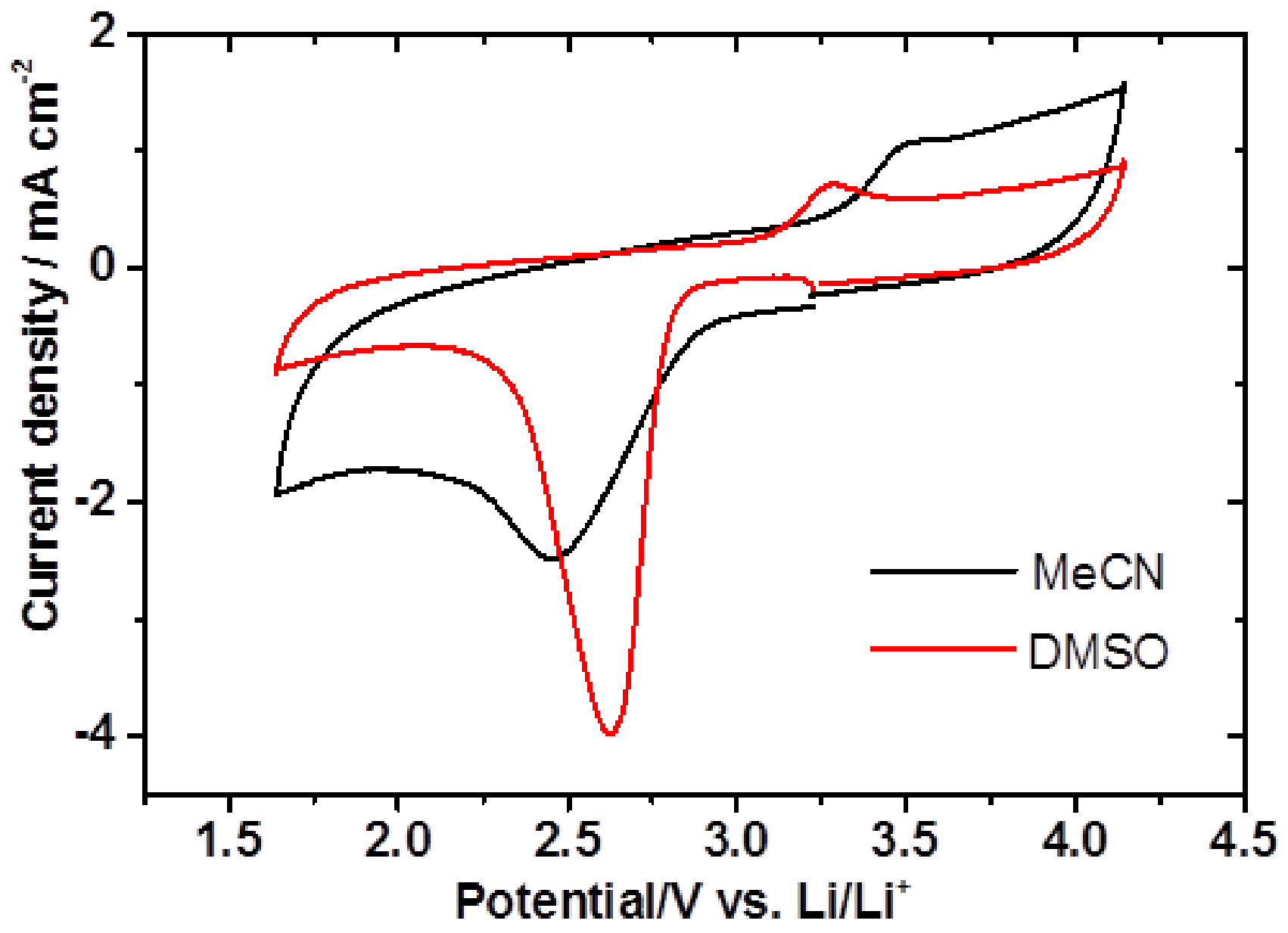

Figure S10 Cyclic voltammograms of $0.5 \mathrm{M} \mathrm{LiClO}_{4} \mathrm{DMSO}$ and $\mathrm{MeCN}$ on GC planar electrode at 10 $\mathrm{mV} / \mathrm{s}$. 
Table S3. Tentative assignment of Raman Peak positions for ORR reaction products detected on glassy carbon.

\begin{tabular}{|c|c|c|}
\hline \multirow{2}{*}{ Reduced dioxygen species } & Peak Position $\left(\mathrm{cm}^{-1}\right)$ & Peak Position $\left(\mathrm{cm}^{-1}\right)$ \\
\hline & $v_{0-0}$ & $\mathrm{vO}_{2}-\mathrm{C}$ \\
\hline $\mathrm{O}_{2}^{-}$ & 1110 & 1520 \\
\hline $\mathrm{LiO}_{2}$ & 1127 & 1500 \\
\hline $\mathrm{HO}_{2}$ & 1179 & 1450 \\
\hline
\end{tabular}




\section{References}

(1) Frens, G.; Controlled Nucleation for the Regulation of the Particle Size in Monodisperse Gold Suspensions. Nature Phys. Sci. 1973, 241, 20-22.

(2) Li, J. F.; Huang, Y. F.; Ding, Y.; Yang, Z. L.; Li, S. B.; Zhou, X. S.; Fan, F. R.; Zhang, W.; Zhou, Z. Y.; Wu, D. Y.; Ren, B.; Wang, Z. L.; Tian, Z. Q. Shell-Isolated Nanoparticle-Enhanced Raman Spectroscopy. Nature. 2010, 464, 392-395.

(3) Aldous, I. M.; Hardwick, L. J. Influence of Tetraalkylammonium Cation Chain Length on Gold and Glassy Carbon Electrode Interfaces for Alkali Metal-Oxygen Batteries. J. Phys. Chem. Lett. 2014, 5, 3924-3930.

(4) Li, J. F.; Tian, X. D.; Li, S. B.; Anema, J. R.; Yang, Z. L.; Ding, Y.; Wu, Y. F.; Zeng, Y. M.; Chen, Q. Z.; Ren, B.; Wang, Z. L.; Tian, Z. Q. Surface Analysis using Shell-Isolated NanoparticleEnhanced Raman Spectroscopy. Nat. Protoc. 2013, 8, 52-65.

(5) Smith, S. R.; Leitch, J. J.; Zhou, C.; Mirza, J.; Li, S. B.; Tian, X. D; Huang, Y. F.; Tian, Z. Q.; Baron, J. Y.; Choi, Y.; Lipkowski, J. Quantitative SHINERS Analysis of Temporal Changes in the Passive Layer at a Gold Electrode Surface in a Thiosulfate Solution. Anal. Chem. 2015, 87, 37913799

(6) Liu, B.; Blaszczyk, A.; Mayor, M.; Wandlowski, T. Redox-Switching in a Viologen-tyep Adlayer: An Electrochemical Shell-Isolated Nanoparticle Enhanced Raman Spectroscopy Study on Au(111)-(1x1) Single Crystal Electrodes. ACS Nano.2011, 7, 5662-5672.

(7) Li J-F, Ding S-Y, Yang Z-L, Bai M-L, Anema JR, Wang X, et al. Extraordinary Enhancement of Raman Scattering from Pyridine on Single Crystal Au and Pt Electrodes by Shell-Isolated $\mathrm{Au}$ Nanoparticles. J. Am. Chem. Soc. 2011, 133, 15922-15925. 